\title{
Magnetic Field Behavior of YBCO step-edge Josephson junctions in rf-washer SQUIDs
}

\author{
M. Bick, J. Schubert, M. Fardmanesh, G. Panaitov, M. Banzet, W. Zander, Y. Zhang and H.-J. Krause
}

\begin{abstract}
The suppression of the critical current in YBCO Josephson junctions by the Earth's magnetic field strongly affects the operation of SQUIDs outside magnetic shielding. Commonly, one observes a modulation of the SQUID fluxvoltage transfer function amplitude, $V_{s q-p p}$, with a period of $\Delta B_{0-v}$ leading to an increased white flux noise level or unstable SQUID operation. Here, we report on the investigation of $\Delta \mathbf{B}_{0-\mathrm{V}}$ of rf-SQUID sensors based on step edge junctions (SEJ) operated in a flip chip configuration with coplanar resonators with integrated flux concentrators. To investigate the origin of the suppression of $V_{\mathrm{sq}-\mathrm{pp}}$, we opened the SQUID loop of some samples and measured the magnetic field dependence of the critical current $I_{\mathrm{e}}(\mathrm{B})$ directly and compared it to $\mathrm{V}_{\mathrm{sq}-\mathrm{pp}}(\mathrm{B})$. It is shown that a junction width in the submicrometer scale is required for operation of the sensors in the Earth's magnetic field.
\end{abstract}

Index Terms - Magnetic field dependence, rf-SQUID, SEJ

\section{INTRODUCTION}

$\mathrm{M}$ ANY SQUID applications require stable sensor operation in unshielded environments. This is especially important for SQUID devices opposed to magnetic field variations of up to $50 \mu \mathrm{T}$ in the Earth's magnetic field which may result in an increased white noise level due to Josephson vortex penetration into the junction [1]-[3]. In the extreme case, the sensors cannot be operated in flux locked loop mode. This is due to an external magnetic field $B$, causing flux penetration into the junction area modulating the critical current, $I_{c}$. This modulation follows a Fraunhofer-like pattern $I_{c}(B)$ with a field period of $\Delta B_{0}$ depending on the width of the junction [4],[5].

To obtain SQUIDs for stable operation in a given magnetic field, it is important to determine the range of the required junction width $w$. For planar junctions, flux focussing effects of the superconducting electrodes lead to a $1 / \mathrm{w}^{2}$ dependence of $\Delta \mathrm{B}_{0}$ [6]. For thick planar devices (wt $>>\lambda_{\mathrm{L}}{ }^{2}$, thick film limit), due to strong demagnetization effects, $\Delta B_{0}=$ $\phi_{0} t /\left(1.2 w^{2} 2 \lambda_{L}\right)$ is predicted where $t$ denotes the film thickness and $\lambda_{L}$ is the London penetration depth. For thin films, the

Manuseript received September 18, 2000.

Marcel Bick, Jürgen Schubert, Grigory Panaitov, Marco Banzet, Willi Zander, Yi Zhang and Hans-Joachim Krause are with the Institut für Schicht - und Ionentechnik, Forschungszentrum Jülich, 52425 Jülich, Germany (corresponding author: telephone +492461613522, email m.bick@fz-juelich.de).

Mehdi Fardmanesh is with the faculty of Electrical and Electronics Engeneering Department, Bilkent University, Ankara, Turkey and presently guest scientist at Forschungszentrum Jülich, Germany. magnetic field penetrates the film almost uniformly and the Meissner screening currents are neglected. The equation $\Delta \mathrm{B}_{0}=1.84 \phi_{0} / \mathrm{w}^{2}$ has been derived for planar grain boundary junctions (GBJ) [6] and verified for SEJs [7],[8]. These formulae only apply to isolated junctions. In the case of washer-SQUIDs, additional flux focussing areas have to be taken into account when calculating $\Delta \mathrm{B}_{0}$. Assuming a homogeneous field distribution in the inner square hole of dimension, d, of an rf washer SQUID with an effective area, $A_{e f f}$, the magnetic field at the first critical current minimum is:

$\Delta \mathrm{B}_{0}=1.84 \phi_{0} \mathrm{~d}^{2} /\left(\mathrm{w}^{2} \mathrm{~A}_{\mathrm{eff}}\right)$

in the thin film limit and in accordance to [3]. The corresponding expression for the thick film limit is:

$\Delta \mathrm{B}_{0}=\phi_{0} \mathrm{t} \mathrm{d}^{2} /\left(1.2 \mathrm{w}^{2} 2 \lambda_{\mathrm{L}} \mathrm{A}_{\mathrm{eff}}\right)$

By using (1) or (2), the optimum junction width for stable operation of SQUIDs in external magnetic fields, $B<\Delta \Delta B_{0}$, can be calculated. Larger effective areas of highly sensitive sensors will automatically lead to smaller field periods, $\Delta \mathrm{B}_{0}$. Therefore, to achieve stable operation in magnetic fields, the junction width has to be small [2], [3].

The stability of the amplitude of the flux-voltage transfer function, $V_{\text {sq-ppy }}$, is the direct criterion for stable SQUID operation. A suppression of $I_{c}$ leads to a reduction of the parameter $\beta_{L}=2 \pi \mathrm{LI}_{c} / \phi_{0}$ (rf-SQUIDs), where $\mathrm{L}$ is the SQUID inductance. $V_{\text {sq-pp }}$ has a maximum at about $\beta_{\mathrm{L}}=1$ and for smaller values, $V_{\text {sq-pp }}\left(\beta_{\mathrm{L}}\right)$ is monotonically decreasing [9]. Therefore, in the case of $\beta_{L} \sim 1$, a suppression of $I_{c}$ should also lead to a suppression of $\mathrm{V}_{\mathrm{sq}-\mathrm{pp}}$. However, for a reliable calculation of the optimum junction width using (1) or (2), the correlation between the field period of the critical current, $\Delta \mathrm{B}_{0}$, and the field period of the flux-voltage transfer function, $\Delta \mathrm{B}_{0-\mathrm{V}}$, has to be known. To our knowledge, this correlation has not been investigated before in detail for SEJ rf-washer SQUIDs.

In this paper, we report experimental investigations on the influence of external magnetic fields on $V_{s q-p p}$ depending on the junction width of SEJ rf-washer SQUIDs. The width dependence of $\Delta \mathrm{B}_{0 . \mathrm{V}}$ is compared to different model predictions. Finally, the suppression of $I_{c}$ will be correlated to the suppression of $V_{\text {sq-pp }}$ by direct measurements of $I_{c}, B_{L}$, and $I_{c}(B)$ using samples with opened SQUID loops.

\section{SAMPLE PREPARATION AND MEASUREMENT SETUP}

Washer-rf-SQUIDs based on step-edge junctions using YBCO films with $\mathrm{t}=200 \mathrm{~nm}$ were fabricated on $\mathrm{LaAlO}_{3}$ 
substrates with the pulsed laser deposition technique [10]. With a $3.5 \mathrm{~mm}$ washer diameter, the magnetometers have a loop of $150 \times 150 \mu \mathrm{m}^{2}(\mathrm{~L} \sim 225 \mathrm{pH})$ and an effective area of $\sim 0.31 \mathrm{~mm}^{2}$. Using conventional photolithography and wet chemical [11] or ion beam etching processes, junction widths of $3 \mu \mathrm{m}$ down to the submicrometer scale were fabricated. The sensors showed a typical magnetic flux noise between 35 and $15 \mu \phi_{0} / \sqrt{ } \mathrm{Hz}$ in the white noise regime. To investigate the period of the magnetic field dependence $\Delta \mathrm{B}_{0-\mathrm{v}}$ of the SQUIDs, $V_{\text {sq-pp }}$ was measured at the output of the SQUID readout electronics as a function of an external magnetic field applied to the zero field cooled (ZFC) SQUIDs at 77K. The field was produced by a calibrated solenoid. To check the influence of different effective sensor areas on the parameter $\Delta \mathrm{B}_{0 . \mathrm{v}}$, the SQUIDs were also operated in flip chip configuration with two different coplanar resonators with integrated flux concentrators [12], with either a $8 \mathrm{~mm}$ rectangular (SR8) or a $13.4 \mathrm{~mm}$ circular (SR13) shape. Investigating the dependence of $\Delta \mathrm{B}_{0-\mathrm{v}}$ on $\mathrm{w}, 24$ SQUIDs with different junction widths were characterized with the same setup. All measurements were performed inside a 3-layer mumetal shield.

To find the correlation between the field dependence of the critical current and the flux-voltage transfer function, $I_{c}(B)$ and $V_{\text {sq-po }}(B)$, respectively, four-probe current-voltage (I-V) measurements on 3 of the 24 already characterized SQUIDs were performed. For this purpose, the SQUID washers were opened mechanically with a diamond cutter. In order not to change the junction properties, processes like photolithography to open the loop and gold evaporation of contact pads were avoided. Instead, gold wires were bonded directly onto the YBCO surface yielding contact resistances of $<5 \Omega$ at $77 \mathrm{~K}$. To check the stability of the junction properties, one sample was bonded but only partly scratched without opening the loop. Measurements of flux noise and $\Delta B_{0-v}$ showed no difference before and after this process. The ZFC SQUID samples with opened loops were characterized in a mu-metal shield with a setup allowing measurements at temperatures down to about $5 \mathrm{~K}$. $\mathrm{I}_{c}(\mathrm{~B})$ was measured in a magnetic field normal to the film surface using a $30 \mu \mathrm{V}$ criterion. To determine $I_{c}$ and $\beta_{L}$ in the presence of large thermal fluctuations $(\Gamma>1)$ at $T=77 \mathrm{~K}$, measurements of the differential resistance, $\mathrm{R}_{\mathrm{d}}=\mathrm{dV} / \mathrm{dI}$, were performed with standard lock-in technique. As described in [13], $I_{c}$ can be derived from the analytic expression of the normalized differential resistance $r_{d}(i)=1-\left\{1 /\left[2\left(i^{2}+\Gamma^{2}\right)\right]-i^{2} /\left(i^{2}+\Gamma^{2}\right){ }^{2}\right\}$ at zero bias current where $\mathrm{i}=\mathrm{I} / \mathrm{I}_{\mathrm{c}}$ denotes the normalized current and $\Gamma=2 \pi \mathrm{k}_{\mathrm{B}} \mathrm{T} /\left(\mathrm{I}_{\mathrm{c}} \phi_{0}\right)$ is the junction noise parameter.

\section{RESUlTS AND DISCUSSION}

The magnetic field dependence of the transfer function $\mathrm{V}_{\mathrm{sq}-\mathrm{pp}}$ of all sensors showed one main maximum enabling determination of the value of the magnetic field at the first minimum: $\Delta \mathrm{B}_{0 . \mathrm{v}}$. For about $30 \%$ of the devices, a Fraunhofer-like dependence of $\mathrm{V}_{\text {sq-pp }}(\mathrm{B})$ was observed. A typical example is shown in Fig. 1. Strongly irregular minimum spacings or even no side minima were found for the other sensors. This behavior and also the observed nonzero minima may be attributed to a nonhomogeneous current distribution in the junction [2],[4],[5] or to flux penetrating into a second grain boundary in series with the main junction as shown in [1] for bicrystal rf-SQUIDs.

Fig. 1 also illustrates the effect of different sensor areas on the field period $\Delta \mathrm{B}_{0 . \mathrm{V}}$. As expected, coplanar resonators with larger effective areas have no influence on the shape of the Fraunhofer-like pattern but reduce the field period following a $1 / A_{\text {eff }}$ dependence according to (1). This is shown in the inset of Fig. 1. When the magnetic field dependence of the washer-SQUID is known, $\Delta B_{0-v}$ can be calculated for every type of flip chip configuration.

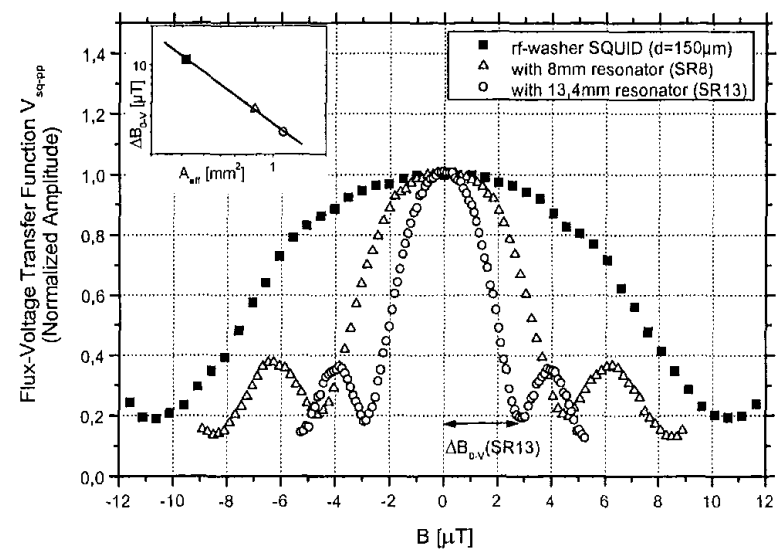

Fig. 1. Magnetic field dependence of $\mathrm{V}_{\mathrm{sq} \text {-p }}$ of an rf-washer SQUID with $w=3 \mu \mathrm{m}$, and effect of coplanar resonators with flux concentrators of two effective sensor areas, $0.78 \mathrm{~mm}^{2}$ and $1.11 \mathrm{~mm}^{2}$. The inset shows the $1 / A_{\mathrm{eff}}$ dependence of $\Delta \mathrm{B}_{0 \mathrm{v}}$ (solid line) compared to the experimental data.

\section{A. Dependence of $\triangle B_{0}$ on the Junction Width w}

To investigate the influence of the junction width on the field period $\Delta \mathrm{B}_{0-\mathrm{v}}$, SQUIDs with different values of $\mathrm{w}$ were studied. In Fig. 2 , the measured $\Delta \mathrm{B}_{0-\mathrm{v}}(\mathrm{w})$ dependence of single washer-SQUIDs without coplanar resonators (symbols) is shown in comparison with model predictions (lines). A clear $1 / \mathrm{w}^{2}$-behavior is observed as predicted in [6] for planar devices. The model for thin films was confirmed for isolated SEJs with $\mathrm{t}=200 \mathrm{~nm}$ similar to those used in our SQUIDs [7] (dashed-dotted line in Fig. 2). However, the observed field enhancement at the junction is larger than expected within the thin film model even when taking into account the flux focussing effect of the SQUID washer according to (1). In our measurements, the majority of data points is lying in the intermediate zone of thin and thick film limit described by (1) and (2) (thick solid and dashed line in Fig. 2). This may be explained by the fact that (1) and (2) are only exact in the extreme thin and thick film limit. Moreover, the derivation of (1) is based on the assumption of a homogeneous flux density in the SQUID hole. Based on the current distribution in a circular washer close to its inner hole, Sloggett et al. [3] derived a simplified expression for the estimation of the flux density, $\mathrm{B}_{\mathrm{ji}}(\xi)$, for points in the hole of a circular washer-SQUID close to the edge, i.e. close to the 
Josephson junction $\left(\mathrm{r}_{\mathrm{i}}-\sigma<<\mathrm{r}_{\mathrm{i}}\right)$. The expression is $B_{j j}(\xi)=B_{m}\left[r_{i} /(8 \xi)\right]^{0.5}$ where $B_{m}$ is the mean value of the flux density, $\sigma$ denotes the radial distance $\left(\sigma<\mathrm{r}_{i}\right), \mathrm{r}_{\mathrm{i}}$ is the inner hole radius, and $\xi=\mathrm{r}_{i}-\sigma$ is the distance from the loop edge [3]. They found the flux density in the middle of the hole to be half of its mean value $B_{m}$ and a field enhancement $\gg>1$ at the edge of the hole where the junction is situated. This principle tendency of a field enhancement $B_{j j} / B_{m}$ at the junction is unlikely to change for SQUIDs with square holes and may be

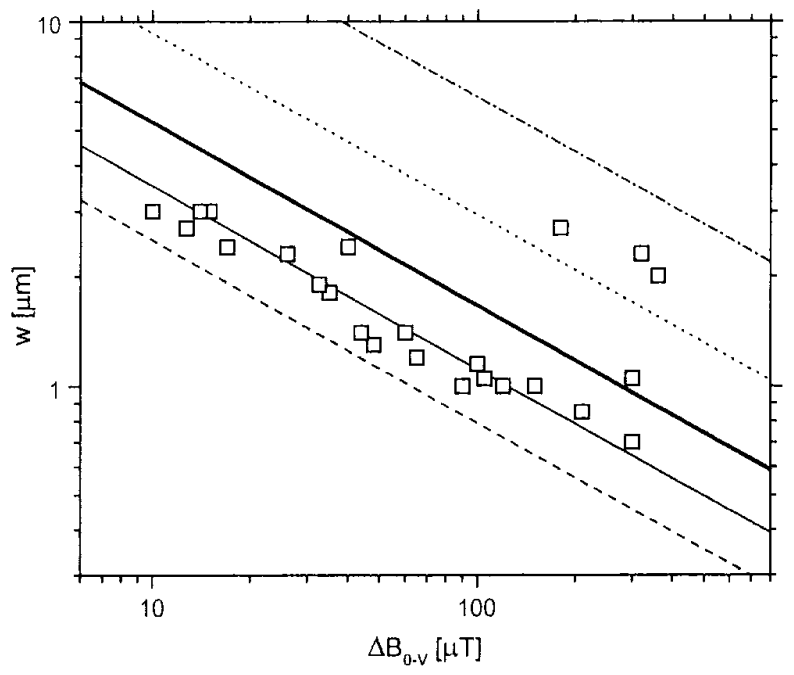

Fig. 2. Dependence of the field period $\Delta \mathrm{B}_{0-\mathrm{v}}$ on the junction width: comparison of our experimental data for single SQUIDs (symbols) with different model predictions for $\mathrm{t}=200 \mathrm{~nm}, \lambda_{\mathrm{L}}=200 \mathrm{~nm}$ at $77 \mathrm{~K}, \mathrm{~d}=150 \mu \mathrm{m}$ and $\mathrm{A}_{\mathrm{c}}=0.31 \mathrm{~mm}^{2}: \Delta \mathrm{B}_{0}=1.84 \phi_{0} / \mathrm{w}^{2}$ (dashed-dotted line: isolated GBJ, thin film limit), $\Delta B_{0}=\phi_{0} t /\left(1.2 \mathrm{w}^{2} 2 \lambda_{\mathrm{L}}\right)$ (dotted line: isolated junction, thick film limit), $\Delta B_{0}=1.84 \phi_{0} d^{2} /\left(w^{2} A_{e f f}\right)$ (thick solid line: SQUID, thin film limit), $\Delta B_{0}=1.84 \phi_{0} d^{2}\left(B_{a r} / B_{i j}\right) /\left(w^{2} A_{e f f}\right)$ (thin solid line: SQUID, thin film limit, taking into account field enhancement of $\left.B_{\mathrm{jj}} / \mathrm{B}_{\mathrm{m}}=2\right)$, and $\Delta \mathrm{B}_{0}=\phi_{0} \mathrm{t} \mathrm{d}^{2}$ / $\left(1.2 w^{2} 2 \lambda_{L} A_{\mathfrak{c r r}}\right)$ (dashed line: SQUID, thick film limit).

the reason for the observed behavior. $A$ fit of our experimental data using $\Delta \mathrm{B}_{0}=1.84 \phi_{0} \mathrm{~d}^{2}\left(\mathrm{~B}_{\mathrm{m}} / \mathrm{B}_{\mathrm{jj}}\right) /\left(\mathrm{w}^{2} \mathrm{~A}_{\text {eff }}\right)$ shows that a value of $B_{j j} / B_{m} \sim 2$ (thin solid line in Fig. 2) is consistent with most of our samples. This enables us to estimate the necessary junction width for our rf-SQUIDs with coplanar resonators for operation in the Earth's magnetic field $\left(B_{\text {earth }}\right.$ $\sim 50 \mu \mathrm{T}$ ). A field period $\Delta \mathrm{B}_{0 . \mathrm{y}}$ of $\sim 100 \mu \mathrm{T}$ is necessary [3] in order not to significantly decrease the transfer function $V_{\text {sq-pp }}$, leading to an increased white noise level of the sensors. Taking into account the effective areas of the different sensor types, we obtain optimum junction widths of $w \sim 0.6$ to $1.2 \mu \mathrm{m}$. 'Magnetically stable' sensors can also be obtained accidentally at larger widths, as shown in Fig. 2 for some samples. This property can be attributed to a strong difference between geometric and effective junction width and has been observed by other groups before [2],[3]. However, for the reproducibility of stable devices, the fabrication of narrow junctions is essential.

\section{B. Correlation of $\Delta \mathrm{B}_{0}$ and $\Delta \mathrm{B}_{0-\mathrm{V}}$}

The analysis of the parameter $\Delta B_{0-v}$ in Section IIA - the period of the magnetic field dependence of the flux-tovoltage transfer function amplitude - was performed on the basis of (1) and (2). As pointed out in Section I, this approach is not strictly correct because these equations are for the period $\Delta \mathrm{B}_{0}$ of the magnetic field dependence of the junction's critical current $I_{c}(B)$. Therefore, in this section, the conformity of $\Delta \mathrm{B}_{0}$ and $\Delta \mathrm{B}_{0-\mathrm{V}}$ for SEJ-rf-SQUIDs will be checked. To exclude uncertainties in this analysis arising from strong junction inhomogenities, only samples with $V_{s q-p p}(B)$ curves showing no strong deviation from a Fraunhofer-like pattern were chosen for the investigation. In this case, the magnetic field behavior is dominated by the critical current of the main junction or by subjunctions in series with a similar field dependence.

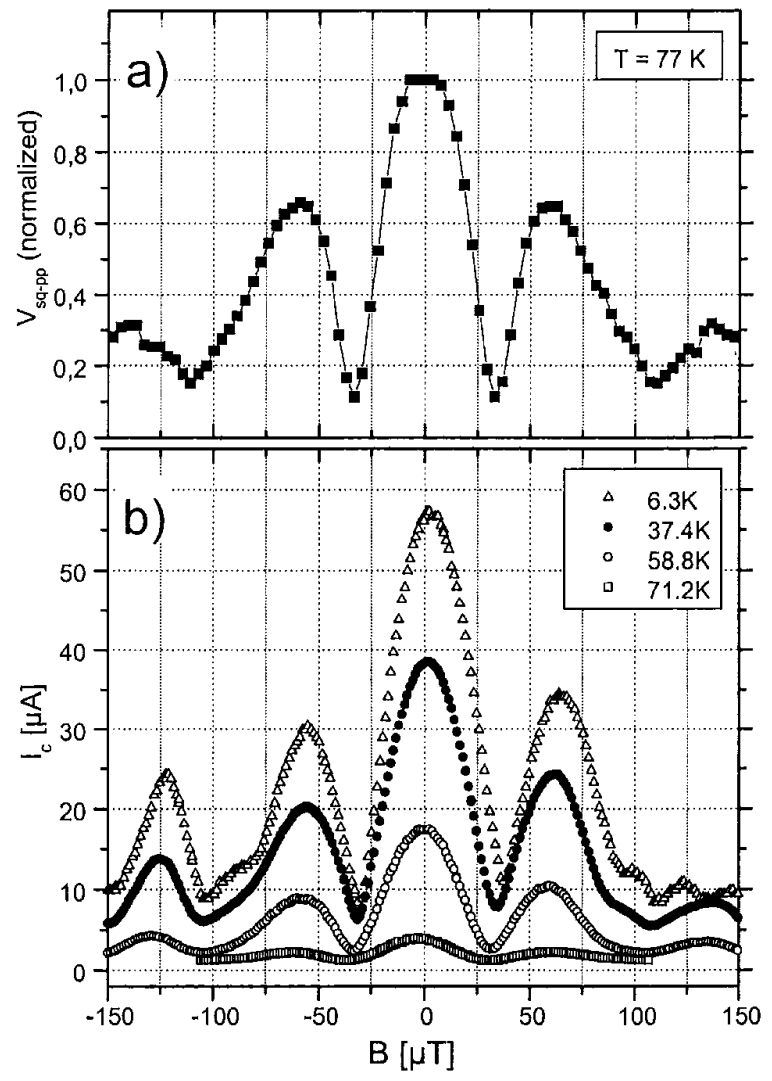

Fig. 3. Comparison of the field dependence of the transfer function $V_{s q-p p}$ of an rf-washer-SQUID and of its critical current $I_{c}$, respectively: a) $V_{\text {sq-pp }}(B)$ at $77 \mathrm{~K}, \mathrm{~b})$ temperature dependent 4-probe measurements of $I_{c}(B)$ on the same SQUID after opening the loop.

In Fig. 3, the $\mathrm{V}_{\mathrm{sq}-\mathrm{pp}}(\mathrm{B})$ dependence of a SQUID is shown in comparison with four-probe measurements of $\mathrm{I}_{c}(\mathrm{~B})$ of the same SQUID after opening the loop. The periods of the Fraunhofer-like patterns $-\Delta \mathrm{B}_{0}$ and $\Delta \mathrm{B}_{0-\mathrm{V}}$, respectively - show reasonably good agreement including a good correlation of the side minima. A similar behavior was found for other devices as well. 
The measurements of $I_{c}(B)$ at different temperatures (Fig. 3b) demonstrate that there is no strong temperature dependence of the period $\Delta B_{0}$. Therefore, the $I_{c}(B)$ curves were acquired at low temperatures to reduce measuring errors as the determination of $I_{c}$ using a voltage criterion is difficult in the presence of high thermal fluctuations at $77 \mathrm{~K}$ for $\mathrm{I}_{\mathrm{c}}$ values of a few $\mu \mathrm{A}$.

All investigated SQUID junctions showed resistively shunted junction (RSJ) behavior. $I_{c}$ was obtained at $77 \mathrm{~K}$ by fitting the junction noise parameter $\Gamma=2 \pi \mathrm{k}_{\mathrm{B}} \mathrm{T} /\left(\mathrm{I}_{\mathrm{c}} \phi_{0}\right)$ to the measurements of the differential resistance (see example in Fig. 4). From $I_{c}, \beta_{L}$ was determined to be $<1.5$ at $77 \mathrm{~K}$ for all samples. A list summarizing the investigated parameters of the samples is given in Table I.

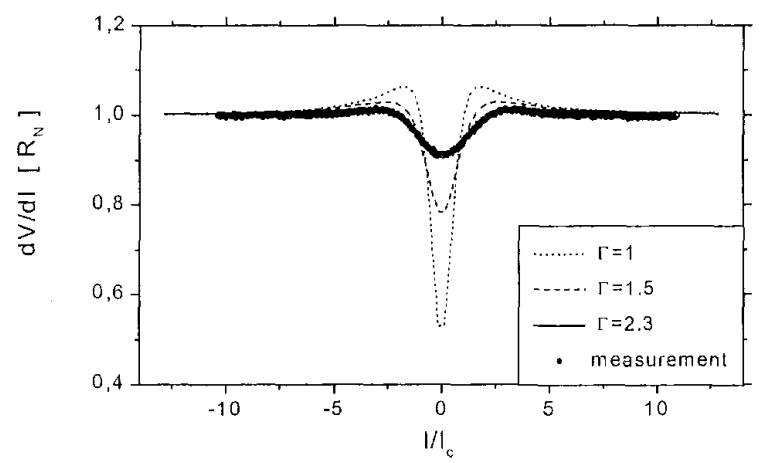

Fig. 4. dV/dI-measurement at $77 \mathrm{~K}$ (sample JS1712) to determine $\beta_{\mathrm{L}}$ : The measurement is in good accordance with the calculation of $\mathrm{dV} / \mathrm{dI}$ on the basis of the RS.J model [13] for $\Gamma=2.3$.

TABLE I

COMPARISON OF SQUID PARAMETERS

\begin{tabular}{lccccc}
\hline Sample & $w[\mu \mathrm{m}]$ & $\begin{array}{r}\mathrm{I}_{\mathrm{c}}[\mu \mathrm{A}] \\
\text { at } 77 \mathrm{~K}\end{array}$ & $\begin{array}{c}\mathrm{B}_{\mathrm{L}} \\
\text { at } 77 \mathrm{~K}\end{array}$ & $\begin{array}{c}\Delta \mathrm{B}_{\text {o-v }}[\mu \mathrm{T}] \\
\mathrm{V}_{\text {sq-pp }}(\mathrm{B})\end{array}$ & $\begin{array}{c}\Delta \mathrm{B}_{0}[\mu \mathrm{T}] \\
\mathrm{I}_{\mathrm{c}}(\mathrm{B})\end{array}$ \\
\hline JSA12 & 2.7 & 2.1 & 1.4 & 12.7 & 14.2 \\
JSA13 & 1.9 & 1.3 & 0.9 & 32.6 & 32.8 \\
JS1712 & 1.4 & 1.4 & 1.0 & 59.5 & 56.0 \\
\hline
\end{tabular}

The overall good agreement between $\Delta \mathrm{B}_{0}$ and $\Delta \mathrm{B}_{0 \mathrm{v}}$ in the case of the 3 investigated samples is confirmed by the fact that the values for $\beta_{\mathrm{L}}$ are not much larger than $\sim 1$ (Table I). In this case, a reduction of $\beta_{L}$ caused by the suppression of the critical current due to an external magnetic field will also

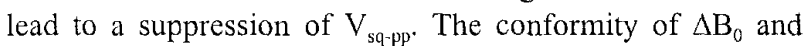
$\Delta B_{0-v}$ was proven indirectly by the determination of $\beta_{L}(<1.5)$ and directly by comparing the measured magnetic field dependences of $I_{c}$ and $V_{\text {sq-pp }}$ (Fig. 4). We conclude that the equations described in the previous section can be applied to yield an estimation for the expected field period $\Delta \mathrm{B}_{0-\mathrm{V}}$ for different junction widths of our SQUID devices.

\section{CONCLUSION}

We have investigated the magnetic field dependence of the flux-voltage transfer function, $V_{\text {sq-pp }}(B)$, for SEJ rf-washer SQUIDs and coplanar resonators with different effective sensor areas and junction widths. The suppression of $\mathrm{V}_{\text {sq-pp }}$ by a magnetic field - commonly leading to an increased white flux noise level - was correlated to a suppression of the critical current in the rf-SQUID junction. This was shown for devices having a reasonable homogeneous current distribution according to their Fraunhofer-like pattern. The width dependence of the field period $\Delta \mathrm{B}_{0-\mathrm{v}}$ of the sensors was compared to different models taking into account the field enhancement at the junction due to the focussing washers. The measured field period, $\Delta \mathrm{B}_{0 . \mathrm{v}}$, scales with $1 / \mathrm{w}^{2}$ as expected for planar devices. It was shown that, due to the large effective areas of SQUIDs with coplanar resonators, junction widths in the submicrometer scale are required for operation in the Earth's magnetic field. Future work will include a detailed characterization of the magnetic field behavior of isolated SEJs and their correlation to these results.

\section{ACKNOWLEDGMENT}

The authors would like to thank P. Selders and R. Akram for technical assistance. Special thanks to P. David for invaluable helpful discussions and for critically reading of the manuscript.

\section{REFERENCES}

[1] E.Il'ichev, V.Schultze, R.P.J.ljsselsteijn, R.Stolz, V.Zakosarenko, H.E.Hoenig, H.-G.Meyer and M.Siegel, "Pecularities of rf-SQUID response in finite magnetic fields", Physica $C$, vol. 330, pp. 155-159, 2000

[2] V.N.Glyantsev, Y.Tavrin, W.Zander, J.Schubert and M.Siegel, "The stability of $\mathrm{dc}$ and rf-SQUIDs in static ambient fields", Supercond.Sci.Technol., vol. 9, pp. A106-108, 1996.

[3] G.J.Sloggett, C.P.Foley, S.Lam, R.A.Binks, and D.L.Dart, "77 K SQUIDs operating in the Earth's magnetic field", IEEE Trans.Appl. Supercond., vol. 7, pp. 3044-3047, 1997.

[4] A.Barone, G.Paterno, "Physics and applications of the Josephson effect", Wiley Interscience, 1982.

[5] R.G.Humphreys and J.A.Edwards, " $\mathrm{YBa}_{2} \mathrm{Cu}_{3} \mathrm{O}_{7}$ thin film grain boundary junctions in a perpendicular magnetic field", Physica $C$, vol. 210 , pp. $42-54,1993$.

[6] P.A.Rosenthal, M.R.Beasley, K.Char, M.S.Colclough and G.Zaharchuk, "Flux focusing effects in planar thin-film grain-boundary Josephson junctions", Appl.Phys.Lett., vol. 59, pp. 3482-3484, 1991.

[7] M.Vaupel, G.Ockenfuss, and R.Wördenweber, "Magnetic field behaviour of small sputtered step-edge junctions", Appl.Phys.Lett., vol. 68, pp. 3623-3625, 1996

[8] E.E.Mitchell, C.P.Foley, K.-H.Mueller, K.E.Leslie, "Vortex penetration and hysteretic behaviour of narrow planar Josephson junctions in a magnetic field", Physica C, vol. 321, pp. 219-230, 1999.

[9] C.M.Falco, and W.H.Parker, "Operation characteristics of thin-film rfbiased SQUIDs", J.Appl.Phys., vol. 46, pp. 3238-3245, 1975.

[10]M.Fardmanesh, J.Schubert, R.Akram, M.Bick, Y.Zhang, M.Banzet, W.Zander, M.Schilling and H.Burkhart, "1/f Noise Characteristics of SEJ YBCO rf-SQUIDs on $\mathrm{LaAlO}_{3}$ Substrate and the Step Structure, Film, and Temperature Dependence", IEEE Trans.Appl.Supercond., submitted for publication.

[11]F.M.Kamm, A.Plettl and P.Ziemann, "Superconducting nanobridges prepared by a self-limiting wet chemical etching process", Supercond. Sci. Technol., vol. 11, pp. 1397-1400, 1998.

[12] Y.Zhang, N.Wolters, X.H.Zeng, J.Schubert, W.Zander, H.Soltner, H.R.Yi, M.Banzet, F.Rüders and A.I.Braginski, "Washer rf-SQUID magnetometers with coplanar resonators at 77K", Appl.Supercond. vol. 6 , pp. 385-390, 1998.

[13]X.H.Zeng, Y.Zhang, B.Chesca, K.Barthel, Ya.S.Greenberg, and A.I.Braginski, "Experimental study of the amplitude-frequency characteristics of HTS rf-SQUIDs", J.Appl.Phys., vol. 88, pp. 6781-6787, 2000 . 